\title{
Effect of upright and ambulant positions versus lying down during the active first stage of labor on birth outcomes among nulliparous women: randomized controlled clinical trial
}

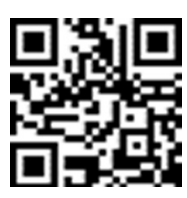

Original article

\author{
Heba Abdel-Fatah Ibrahimª,b, * Hanan Ibrahim Ibrahim Said ${ }^{c}$, Wafaa Taha Ibrahim Elgzar ${ }^{b, c}$ \\ aObstetrics and Woman Health Nursing department, Benha University, Benha, Kalubia 13741, Egypt \\ ${ }^{b}$ Maternity and Childhood Nursing Department, Nursing College, Najran University, Najran 66251, Kingdom of Saudi Arabia \\ cObstetrics and Gynecologic Nursing department, Damanhour University, Damanhour 22511, Egypt
}

Received: 12 December 2019; Accepted: 15 January 2020; Published: 20 September 2020

Abstract: Objective: To examine the effect of upright and ambulant positions versus lying down during the active first stage of labor on birth outcomes for nulliparous women.

Methods: This is a randomized controlled clinical trial conducted at the delivery department of Damanhour Educational Institute, El Behira Governorate, Egypt. The study sample involved 150 parturient women equally divided into intervention and control groups using randomization block technique. The researchers used four tools for data collection: Demographic data interview schedule, World Health Organization Partograph, Apgar's score, to evaluate neonatal outcomes, and visual analogue pain intensity scale. For the study group, the parturient women were encouraged to assume one of the upright positions or ambulating around the bed so as to maintain the pelvis in vertical plane as far as possible for $20-25 \mathrm{~min}$ for every $1 \mathrm{~h}$. The control group received routine hospital care, which includes lying down in bed. IBM SPSS 23.0 was used to analyze the data.

Results: Significant differences $(P<0.05)$ were observed between the study and control groups in relation to cervical dilation, fetal head descent, uterine contractions interval, and frequency. The duration of the first stage of labor significantly reduced $(P=0.018)$ in the intervention group compared with control group. No significant differences $(P>0.05)$ were observed between both groups in term of emergency cesarean birth rates, oxytocin use, and neonatal outcomes.

Conclusions: This study proves that upright and ambulant positions significantly enhance uterine contractility, cervical dilatation, and fetal head descent and reduce the first stage duration.

Keywords: birth outcomes $\bullet$ first stage of labor • lying down • upright position

(c) Shanxi Medical Periodical Press.

How to cite this article: Ibrahim HA, Said HII, Elgzar WTI. Effect of upright and ambulant positions versus lying down during the active first stage of labor on birth outcomes among nulliparous women: randomized controlled clinical trial. Front Nurs. 2020;3:239-248. 


\section{Introduction}

Although labor is a normal physiological process, still it is a dynamic and complex process with many physiological and psychological variables. ${ }^{1}$ However, the care provided to women during childbirth has the potential to affect them both physiologically and psychologically. Similarly, short- and long-term delivery management are the most common medical problems facing health care providers. Surely, normal progression of labor is closely related to a proper compatible management. Improper labor management may lead to prolonged and/or obstructed labor, which may lead to maternal exhaustion, rupture of the uterus, postpartum bleeding, and infection. Thus, prevention and early detection of any deviation from the normal would dramatically reduce the incidence of dystocia and its adverse consequence..$^{2,3}$

Dystocia (difficult childbirth) is characterized by abnormal slow labor progression due to ineffective uterine contractions, fetal malpresentation or malposition, and inadequate pelvic capacity or pelvic floor defect. Evidence points out that up to one-third of nulliparous women are subjected to a delay in the first stage of labor. ${ }^{4,5}$ The prevalence of dystocia ranges from $4.8 \%$ to $21 \%$ among vaginal deliveries. According to the American College of Obstetricians and Gynecologists, about $60 \%$ of cesarean sections (CS) are attributed to dystocia. ${ }^{6}$

As dystocia is the most common cause of cesarean birth, efforts to decrease the rate of cesarean delivery should include efforts to maintain the physiological process in which women's mobility is essential. Women should feel that they can deliver successfully with satisfaction in a supportive environment, which might reduce their fear of pain. Health team should support their ability to overcome labor pains, including the freedom to walk, sit, move, and change their position during labor. ${ }^{7}$

Undoubtedly, maternal position during the first stage of labor affects all factors of normal delivery including power, passenger, passage, and psyche. It affects the characteristics and efficiency of uterine contractions, duration of labor, fetal/neonatal well-being, and women comfort. $^{8}$

The recumbent position is the most common position for women during the first stage of labor at health facilities in both developed and developing countries. Although medical staff say that recumbent position is an appropriate position for delivery, there is no scientific confirmation on its advantages to mothers or newborns. Observational researches have indicated that when mothers are lying on their backs during childbirth, it may have adverse effects on uterine contractions and postural hypotension, and decrease placental perfusion. ${ }^{9}$ So the National Institute for Health and Clinical Excellence recommends that parturient should be encouraged to avoid the supine position during labor and delivery. ${ }^{10}$

Adoption of the upright position and ambulation at the first stage of labor are part of the World Health Organization recommendation for the humanization of health care. It suggests that returning to noninvasive procedures avoids unnecessary or additional interventions and ensures maximum health benefits for the women and her newborn. ${ }^{11}$

Congruently, there is clear and significant evidence that upright and ambulant positions in the first stage of labor have several advantages. These include the effect of gravity that may reduce aortic compression, increase the duration, frequency, and intensity of uterine contractions. Furthermore, it may help in better adjustment of the fetus to the birth canal and increase pelvic capacity, which in turn may reduce the need for instrumental deliveries. ${ }^{12}$ In addition, it may decrease the labor length (time), CS incidence, and analgesia requisite. It may assist cervical dilatation, fetal head descent, and increase satisfaction in childbirth experience. From other point of view, it does not appear to be related to increased intervention or negative outcomes on mothers and their newborns. ${ }^{7,9}$

Lawrence et al. conducted a systemic review to evaluate the effects of different upright versus recumbent positions for women on labor duration, mode of delivery, and other essential maternal and neonatal outcomes in the first stage of labor. They found a considerable heterogeneity and high-performance bias in their studies. Accordingly, they suggested to perform better quality trials so as to confirm the true risks and benefits of upright and ambulant positions compared with recumbent positions. ${ }^{9}$ Therefore, it is important that studies that evaluate upright and recumbent positions during the first stage of labor are monitored to ensure good quality data. Such studies can provide strong evidence on whether or not an upright approach improves maternal and neonatal outcomes. ${ }^{13}$ Therefore, further researches are needed to evaluate positive effects as well as any probable negative effects of upright positions in the first stage of labor on maternal and neonatal outcomes. If such data made available, it will increase maternity nurse's base of knowledge regarding evidence-based management during the first stage of labor.

\subsection{Aim of the study}

This study aimed to examine the effect of upright and ambulant position versus lying down during the active phase of the first stage of labor on birth outcomes in nulliparous women. 


\subsection{Research hypotheses}

$(\mathrm{H} 0)$ parturient who was in upright or ambulant positions during the active phase of the first stage of labor exhibits similar birth outcomes than those who were in lying down positions.

(H1) parturient who was in upright or ambulant positions during the active phase of the first stage of labor exhibits more favorable birth outcomes than those who were in lying down positions.

\section{Materials and method}

\subsection{Study design}

This is a randomized controlled clinical trial in which the effect of one independent variable (upright position for nulliparous women) on one dependent variable (birth outcomes) has been investigated.

\subsubsection{Operational definition}

\subsubsection{Birth Outcomes}

In this study, it refers to the duration of the first, second, and third stage of labor, labor progress parameters (uterine contractions interval, duration, frequency, labor pain intensity, cervical dilation, and fetal head descent), mode of birth defined as (spontaneous vaginal delivery, instrumental, and emergency CS), and neonatal condition at birth.

\subsection{The study setting}

This study was conducted at the delivery department at Damanhour Educational Institute affiliated to the ministry of health in El Behira Governorate, Egypt.

\subsection{Participants and sample size}

The study consisted of a convenience sample of 150 parturient in their active first stage of labor based on the Epi-Info program, which was used to estimate the sample size using the following parameters: expected frequency $=50 \%$; acceptable error $=5 \%$; confidence coefficient $=99 \%$; sample size $=150$; and power analysis $=80 \%$. The study subjects joined this study according to the following inclusion criteria: nulliparous women, full-term, singleton pregnancy, normal size fetus with occipito-anterior position, in the active phase of first stage labor, free from any chronic diseases, intended spontaneous vaginal delivery, and willing to participate in the study. Exclusion criteria include any pregnancy complication, short stature, apparent pelvic abnormalities, and current history of a leg injury, fractures, and deep vein thrombosis.

The selected women were assigned to study and control groups using a randomization block technique. The randomization block was performed manually according to six steps. (1) A list of numbers from 1 to 150 was prepared by the researchers. (2) Each number from 1 to 150 was written again in a separate small piece of paper. (3) Each of the small piece of paper was folded until the number became invisible, then all the papers were mixed in a large bowl. (4) The 150 small papers were randomly and blindly divided into eight blocks; the first seven blocks consists of 20 random numbers and the last one has 10 remaining numbers. (5) From each block, $50 \%$ of the numbers was randomly and blindly selected and assigned to the study group and the remaining $50 \%$ to the control group. (6) Finally, the sequence of cases is recorded in the preset list (in front of each number the researcher writes the word "case or control") to be considered during data collection.

The study group (G1) included 75 parturient who assumed upright positions such as (standing, sitting, kneeling, and squatting) or ambulating position for 20-25 min every $1 \mathrm{~h}$. The control group (G2) involved 75 parturient who assumed lying down position as in routine hospital care.

\subsection{Instruments}

Four instruments were used for data collection:

Tool I: Demographic data interview schedule. It includes age, educational qualification, residence, occupation, weight, height, and receiving information regarding labor preparations in addition to the history of the current pregnancy.

Tool II: World Health Organization Partograph ${ }^{14}$. to monitor the progress of labor in term of cervical dilatation, fetal head descent, uterine contraction characteristics (duration, frequency, and interval), and fetal heart rates. All data were measured at baseline and repeated measures at one-hour interval in addition to other birth outcomes such as mode of delivery, oxytocin use, and the duration of each stage of labor.

Tool III: Apgar's score ${ }^{15}$ at first and fifth minute to evaluate the neonatal outcomes.

Tool IV: Visual analogue pain intensity scale: It is a standardized linear scale that was adopted by Mc Caffery and Pasero ${ }^{16}$ and used to evaluate pain severity. It is a self-reported $10 \mathrm{~cm}$ horizontal line representing the subjective estimation of pain 
intensity. It ranged from 0 (no pain) to 10 (worst pain) in between the two extreme points. The line is numbered on equal distances $(1 \mathrm{~cm})$ : mild pain $(1,2)$, moderate $(3,4)$, sever $(5,6)$, very sever $(7,8)$, and worst pain $(9,10)$. Each degree of pain is elaborated with facial expressions of a picture.

All the instruments were tested for content validity by a panel of five specialists from the field of maternity nursing and one expert from biostatistics. The tool reliability was tested using the Cronbach's alpha test. The reliability results were $0.850,0.766$, and 0.832 for Tools II, III, and IV, respectively. A pilot study was performed on $10 \%$ of the study sample to ensure the applicability of the tools.

\subsection{The procedure of data collection}

Data were collected over a period of 5 months from December 2018 to May 2019. The researchers screened all nulliparous parturient in the latent phase of the first stage of labor. Rapid examination for the parturient file was done to ensure her eligibility for the study and for assigning to study or control group according to the predetermined randomization blocks list. Then, they were individually interviewed by the researchers to take oral consent after explaining the study purpose. The researchers collected the baseline data from both groups at the beginning of the active phase such as uterine contraction characteristics (duration, interval, and frequency), cervical dilatation, fetal head descent, and fetal heart rate by using Tool II and evaluation value of the severity of pain by using Tool IV.

For study group, the parturient women were encouraged to assume one of the upright positions as (standing, sitting, kneeling, or squatting) or ambulating around the bed to maintain the pelvis in vertical plane as far as possible for 20-25 min for every $1 \mathrm{~h}$. Then the woman was permitted to lie in the bed in her left lateral position. She was advised to repeat these positions up to full cervical dilatation. The researchers followed the application of the intervention with each parturient until approaching second stage of labor. In contrast, the parturient women in the control group were assessed without intervention with normal routine hospital care, which includes lying down in bed. Therefore, the women in the control group were informed that they should lie on their left or right lateral position and avoid lying on their back to avoid inferior vena cava compression. Informed oral consent was taken from all participants in both groups. Another one replaces women who refuse participation or who want to withdraw from the study so that sample size is maintained.
During that time, the researchers evaluated labor progress every $1 \mathrm{~h}$ in term of uterine contraction characteristics (duration, interval, and frequency), cervical dilatation, fetal head descent, oxytocin used, and fetal heart rate by using Tool II and evaluation of the severity of pain by using Tool IV. The assessment of labor progress stopped when the woman approached the second stage of labor. Moreover, the other birth outcomes were recorded in the term of duration of the first, second, and third stage of labor and mode of delivery. Immediately after birth, the neonatal outcomes such as baby birth, weight, and head circumference were registered in term of Apgar's score at first and fifth minute using Tool III.

\subsection{Statistical analysis}

Statistical Package for the Social Science (SPSS) version 23.0 was used for data analysis. The statistical significance set at $P<0.05$. Descriptive statistics, as mean and standard deviation, were used for reporting normally distributed numerical variables where as numbers and percentages were used to describe categorical variables. The differences of categorical variables (sociodemographic variables, duration of each stage of labor, and admission of Neonatal Intensive Care Unit (NICU)) between the study and control groups were assessed using the chi-squared test or Fisher's exact test. Comparisons of labor progress parameters among the two groups at each time point were compared using repeated ANOVA and independent sample $t$-test.

\subsection{Ethical consideration}

The researchers were committed to ethical rules at all study stages. An official letter consent letter was given to Damanhour Educational Institute by Damanhour University after getting the clarifications about the study aim. Then informed oral consent was obtained from the study participants and they were informed that their contribution to the study was elective and they have the right to withdraw from the study at any time without any consequences.

\section{Results}

\subsection{Description of study subjects}

The demographic characteristics of the study participants $(n=150)$ were explained in Table 1 . No significant differences $(P>0.05)$ are shown between the study and control groups in term of age, education, working status, residence, and BMI. Likewise, no significant difference $(P>0.05)$ in the gestational age as the gestational age 


\begin{tabular}{|c|c|c|c|c|c|c|}
\hline \multirow[t]{2}{*}{ Items } & \multicolumn{2}{|c|}{ Study group } & \multicolumn{2}{|c|}{ Control group } & \multirow[t]{2}{*}{ Significant test } & \multirow[t]{2}{*}{$P$-value } \\
\hline & $n=75$ & $\%$ & $n=75$ & $\%$ & & \\
\hline \multicolumn{7}{|l|}{ Age (years) } \\
\hline$\leq 20$ & 8 & 10.7 & 6 & 8.0 & & \\
\hline $21-34$ & 60 & 80.0 & 67 & 89.3 & $3.347^{b}$ & 0.164 \\
\hline$\geq 35$ & 7 & 9.3 & 2 & 2.7 & & \\
\hline \multicolumn{7}{|l|}{ Education } \\
\hline Read and write & 17 & 22.7 & 8 & 10.7 & $4.684^{a}$ & 0.096 \\
\hline Secondary & 44 & 58.7 & 46 & 61.3 & & \\
\hline University & 14 & 18.7 & 21 & 28.0 & & \\
\hline \multicolumn{7}{|l|}{ Working status } \\
\hline Working & 18 & 24.0 & 21 & 28.0 & $0.312^{\mathrm{a}}$ & 0.577 \\
\hline House wife & 57 & 76.0 & 54 & 72.0 & & \\
\hline \multicolumn{7}{|l|}{ Residence } \\
\hline Rural & 60 & 80.0 & 54 & 72.0 & $1.316^{\mathrm{a}}$ & 0.251 \\
\hline Urban & 15 & 20.0 & 21 & 28.0 & & \\
\hline$B M I(M \pm S D)$ & $25.62 \pm 2.59$ & & $26.38 \pm 2.30$ & & $0.448^{\circ}$ & 0.655 \\
\hline Gestational weeks $(M \pm S D)$ & $38.37 \pm 0.67$ & & $38.68 \pm 0.98$ & & $0.097^{c}$ & 0.923 \\
\hline
\end{tabular}

Note: ${ }^{C}$ Chi-square test; ${ }^{b}$ Fisher exact test; ' Independent sample $t$-test.

Table 1. Demographic characteristics, BMI, and weeks of gestation of parturient women in the two groups $(n=150)$.

mean of the study group was $38.37 \pm 0.67$ compared with $38.68 \pm 0.98$ of the control group.

\subsection{Birth outcomes}

\subsubsection{Labor progress parameters}

Labor progress parameters in Table 2 indicated no significant differences $(P>0.05)$ in the baseline assessment of uterine contraction, cervical dilation, fetal head descent, and fetal heart rate among the two groups. However, after intervention, significant differences $(P<0.05)$ were observed between the study and control groups in relation to interval and frequency of uterine contractions, cervical dilation, and fetal head descent. This indicates improvement in the cervical dilation progress, and fetal head descent, increasing frequency and decreasing interval between uterine contractions in the study group. On the other hand, no significant differences $(P>0.05)$ were observed between both groups regarding fetal heart rates and pain visual analog scale score at all post-intervention time points.

\subsubsection{Cesarean birth rates, oxytocin use, and the duration of labor}

Table 3 shows that there were no significant differences in the cesarean birth rates, oxytocin use, and the second and third stage of labor duration among the two groups. Upright and ambulant positions in the first stage of labor had no impact on the mode of delivery, oxytocin use, and the duration of the second and third stage of labor. While there was a significant difference $(P=0.018)$ between the study and control groups regarding the duration of the first stage of labor. The mean duration of the first stage was $11.88 \pm 2.50 \mathrm{~h}$ in the study group compared with $12.74 \pm 1.86 \mathrm{~h}$ in the control group.

\subsubsection{Neonatal outcomes}

As shown in Table 4, there were no significant differences $(P>0.05)$ between both groups in relation to neonates' outcomes (baby birth weight, baby head circumference, and Apgar's score at first and fifth minute). Also, in Table 5, the Fisher exact test showed that there was no significant difference $(P=0.467)$ in neonates' ICU admission between both groups.

\section{Discussion}

Evidence-based maternity care focuses on practices that increase maternal and newborn safety. If policymakers and health care team want to improve the quality of obstetric care effectively, it is important that they implement evidence-based clinical practices in routine obstetrics and women health care. ${ }^{17}$ Based upon this view, the current study was conducted using true experimental design to evaluate positive effects as well as any probable negative effects of upright and ambulant positions in the active first 


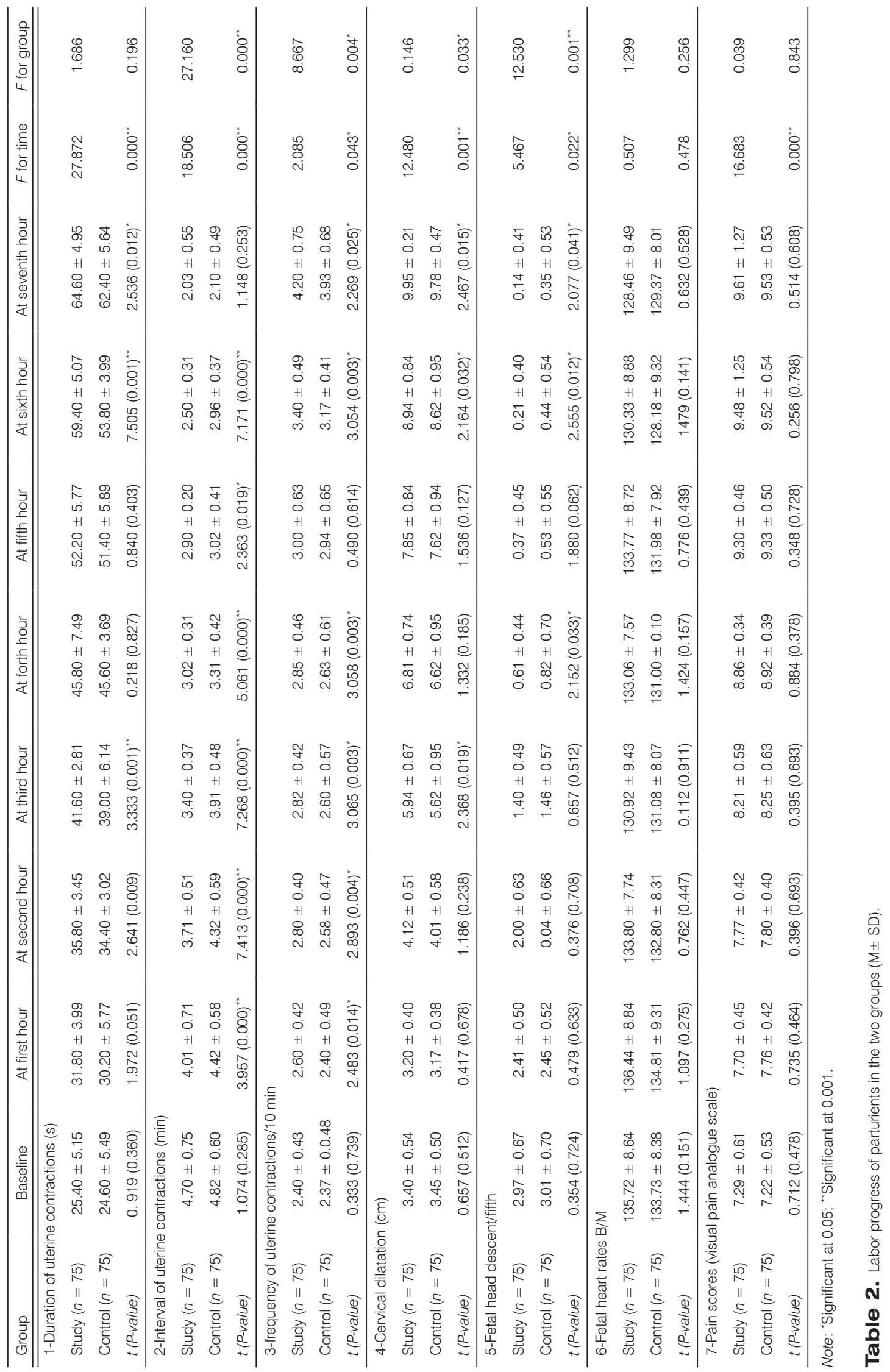




\begin{tabular}{|c|c|c|c|c|c|c|}
\hline Characteristic & \multicolumn{2}{|c|}{ Study group $(n=75)$} & \multicolumn{2}{|c|}{ Control group $(n=75)$} & Significant test & $P$-value \\
\hline \multicolumn{7}{|l|}{ Mode of delivery (cesarean birth rates) } \\
\hline Spontaneous vaginal delivery & 63 & 84.0 & 59 & 78.7 & $0.703^{\mathrm{a}}$ & 0.402 \\
\hline Lower segment cesarean section (LSCS) & 12 & 16.0 & 16 & 21.3 & & \\
\hline \multicolumn{7}{|l|}{ Use of oxytocin augmentation } \\
\hline No & 19 & 25.3 & 13 & 17.3 & $1.430^{\mathrm{a}}$ & 0.232 \\
\hline Yes & 56 & 74.7 & 62 & 82.7 & & \\
\hline \multicolumn{7}{|l|}{ Duration of labor stages } \\
\hline First stage (h) & \multicolumn{2}{|c|}{$11.88 \pm 2.50$} & \multicolumn{2}{|c|}{$12.74 \pm 1.86$} & $2.387^{b}$ & $0.018^{\star}$ \\
\hline Second stage (min) & \multicolumn{2}{|c|}{$62.40 \pm 8.08$} & \multicolumn{2}{|c|}{$65.47 \pm 12.65$} & $1.727^{\mathrm{b}}$ & 0.086 \\
\hline Third stage (min) & \multicolumn{2}{|c|}{$11.53 \pm 2.32$} & \multicolumn{2}{|c|}{$11.77 \pm 3.61$} & $0.478^{b}$ & 0.633 \\
\hline
\end{tabular}

Note: ${ }^{a}$ Chi-square test; ' bIndependent sample $t$ test; *Significant at 0.05.

Table 3. Cesarean birth rates, oxytocin use, and labor stages duration among parturient women in the two groups $(n=150)$.

\begin{tabular}{lcccc}
\hline Characteristic & Study group $(n=75)$ & Control group $(n=75)$ & $t$ & $P$-value \\
\hline \hline Baby birth weight $(\mathrm{kg})$ & $2.96 \pm 0.34$ & $2.92 \pm 0.28$ & 0.773 & 0.441 \\
Baby head circumference $(\mathrm{cm})$ & $33.54 \pm 1.45$ & $33.52 \pm 1.48$ & 0.108 & 0.914 \\
Apgar's score at 1 min & $6.92 \pm 1.36$ & $7.20 \pm 1.20$ & 0.912 & 0.363 \\
Apgar's score at 5 min & $8.98 \pm 1.19$ & $9.28 \pm 1.51$ & 1.189 & 0.236 \\
\hline
\end{tabular}

Note: $t=$ independent sample $t$ test.

Table 4. Neonates' outcome between the two groups $(M \pm S D)$.

\begin{tabular}{lcccccc}
\hline Neonates' admission to NICU & Study group $(n=75)$ & Control group $(n=75)$ & Significant test & $P$-value \\
\hline \hline Yes & 3 & 4.0 & 5 & 6.7 & $0.528^{\text {a }}$ & 0.467 \\
No & 72 & 96.0 & 70 & 93.3 & & \\
\hline
\end{tabular}

Note: aFisher Exact test.

Table 5. Neonates' admission to Neonatal Intensive Care Unit (NICU) $(n=150)$.

stage of labor on labor progress, labor duration, and other maternal, fetal, and neonatal outcomes.

The current study found that upright and ambulant positions increase the frequency of uterine contractions and reduce the interval between contractions in the study group over six-time points out of a total of seventime points compared with the control group. Moreover, the duration of uterine contraction significantly increased in three-time points at third, sixth and seventh hour of intervention. These findings suggested that upright and ambulant positions in the first stage of labor were effective in improving the progress of uterine contractions in nulliparous women. The greater effectiveness of the upright and ambulant positions might be explained by the fact that upright and ambulant positions may benefit from the effect of gravity, which can prevent aortic compression, thus improving the blood supply to the uterus and stimulating the uterine contractions.

Similar effects of assuming upright and ambulant positions in enhancing the uterine contractility have been reported in previous studies conducted by Emam and Al-Zahrani and Mathew et al. The former found a significant improvement in the progress of uterine contractions by increasing its duration and frequency and decreasing the interval between it in the upright group, while the recumbent group shows less progress. The latter had clarified that the ambulation in the first stage of labor results in increase of the quality of uterine contractions. ${ }^{18,19}$

Moreover, upright position and ambulation during active first stage of labor appear to be effective in improving the progress of cervical dilatation, and fetal head descent in the study group compared with control group. This can be attributed to the fact that upright positions allow the gravity to assist in the descent of the fetal head, as well as increase the pelvic capacity giving the baby more room to descend and rotate. Therefore, the direct pressure on the cervix by fetal head leading to improvement in cervical dilatation and progress of labor. ${ }^{20,21}$ These results are consistent with at least three other studies. In the first one, Emam and Al-Zahrani 
indicated a significant difference between upright and recumbent groups in relation to fetal head descent and cervical dilatation progress in favor of the upright group. ${ }^{18}$ In the second study, Hassan had reported a significant improvement in the progress of fetal head descent and cervical dilatation in the experimental group after performing the pelvic rocking exercise using sitting position during the active phase of the first stage of labor as compared with the control group. ${ }^{22}$ In the third study, Lawrence et al. who had done a Cochrane review on 25 studies to evaluate the effect of ambulation and upright positions in the first stage of labor, recognized that upright positions were more effective in the cervical dilation progress than supine position. ${ }^{9}$

On the other hand, Manonmani, who had conducted a true experimental study to evaluate the effect of walking versus laying down in semi fowler's position on birth outcomes among parturient women. He reported no significant difference between walking group and semi fowler's group regarding the cervical dilation rate. ${ }^{23}$ The difference between the current study finding and the latter one may be attributed to the difference in the study subject' parity. The current study was conducted on nulliparous women only whereas Manonmani' study was conducted on nulliparous and multiparous parturient. Parity is found to be positively correlated with the cervical dilation.

In the present study, upright positions and ambulation during the first stage of labor had no significant effect on the fetal heart rates and pain visual analog scale score at all post-intervention time points. In this regard, Miquelutti et al. had conducted a randomized controlled trial to determine the effect of upright position on maternal satisfaction during labor. They reported no significant difference between the study and control groups in relation to visual analog scale score. ${ }^{24}$ In contrast, Gizzo et al. observed a significant difference between the upright and laying down group in term of pain numeric rating scale score and the need for analgesics. ${ }^{12}$ The difference between this study finding and the latter one may be due to the difference in study design. This study was conducted using a true experimental design, while, Gizzo et al. conducted an observational cohort design.

The present study revealed that upright positions and ambulation significantly reduce the duration of the first stage of labor. The probable positive effect of gravity is increasing uterine blood flow and uterine contractions, enhances baby alignment to the birth canal, helps the fetal head descent, and facilitates cervical dilatation. Eventually reducing the first stage duration. Several studies were conducted around the world to assess the effect of upright positions in reducing the first stage duration. Most of these studies are in the same line and confirming that upright positions significantly reduce the first stage duration (Rana and Chopra, Lawrence et al., Ben Regaya et al.)..${ }^{9,25,26}$ Although there was a significant difference between the two groups toward the first stage duration, there were no significant differences observed in the duration of the second and third stages. Also, Lawrence et al. study reported that there were no significant differences between intervention and control groups for the length of the second or third stage of labor. ${ }^{9}$

Although Gau et al. and Taavoni et al. found that upright position and ambulation during the first stage of labor decrease oxytocin use and reduce CS rate, no significant differences were recorded in the current study between both groups in term of CS rate and oxytocin use. ${ }^{27,28}$

Deliktaş and Kabukçuoğlu have found in a recent meta-analysis that the effects of upright position and ambulation on operative delivery were insignificant, which complied with the above findings. ${ }^{29}$ Also, Miquelutt et al. suggested no significant difference among the upright and laying down groups in terms of birth type. ${ }^{24}$ Furthermore, Roberts et al. who conducted a meta-analysis of randomized controlled trials involving a total of 1,161 parturient women. They evaluated the impact of ambulation and upright positions during the first stage of labor on type of delivery and other birth outcomes among parturient women. They reported no significant differences between the upright and recumbent group in oxytocin use and the delivery type. ${ }^{30}$

Moreover, the results of the current study reported no significant differences between both groups in relation to neonates' outcomes, which include neonates' ICU transfer, and Apgar's score at the first and fifth minute. These findings are in agreement with Gizzo et al. and Lawrence et al. The former had found no differences between the upright and recumbent group in terms of neonates' outcomes. The latter reported no significant differences between the intervention and control groups in terms of neonatal Apgar's score at the first and fifth minute..$^{9,12}$ Meanwhile, Ben Regaya et al. had different finding and stated that upright position leads to a net Apgar's score improvement at the first and fifth minute. It also decreased the admission rate to the NICU. ${ }^{26}$

\section{Conclusions}

Based on the overall findings of the current study, $\mathrm{H} 1$ is accepted and $\mathrm{HO}$ is rejected. This study provides clinical evidence on the significant progress of the first stage of labor when assuming upright position and ambulation compared with lying down. This significant progress achieved in terms of enhancing cervical dilatation, fetal had descent, uterine contractions 
frequency, and duration. It also reduced the first stage duration without any adverse effect on maternal, fetal, or neonatal outcomes.

\section{Limitation}

Although the use of the blinded technique would have been ideal for this randomized controlled clinical trial, it was not possible in the current study. The intervention nature and the presence of more than one parturient in the same room represent a practical barrier at time of data collection.

\section{Recommendation}

Maternity nurses and midwives should be trained to support parturient women both in upright and ambulant

\section{References}

1. Hollins Martin CJ, Martin CR. A narrative review of maternal physical activity during labour and its effects upon length of first stage. Complement Ther Clin Pract. 2013;19:44-49.

2. Simposon K, Creehan P. Perinatal Nursing. 4th ed. Philadelphia: Lippincott Williams \&Wilkins; 2014;343-398.

3. Wards S, Hisley SH. Maternal-Child Nursing Care: Optimizing Outcomes for Mothers, Children, and Families. Philadelphia: F.A. Davis Company; 2009:355-342.

4. Ronel D, Wiznitzer A, Sergienko R, Zlotnik A, Sheiner E. Trends, risk factors and pregnancy outcome in women with uterine rupture. Arch Gynecol Obstet. 2012;285:317-321.

5. Kjaergaard H, Olsen J, Ottesen B, Dykes AK. Incidence and outcomes of dystocia in the active phase of labor in term nulliparous women with spontaneous labor onset. Acta Obstet Gynecol Scand. 2009;88:402-407.

6. Cheng YW, Norwitz ER, Caughey AB. The relationship of fetal position and ethnicity with shoulder dystocia and birth injury. Am J Obstet Gynecol. 2006;195:856-862.

7. Ondeck M. Healthy birth practice: walk, move around, and change positions throughout labor. J Perinat Educ. 2014;23:188-193.

8. Abdolahian S, Ghavi F, Abdollahifard S, SheikhanF. Effect of dance labor on the management of active phase labor pain \& clients' satisfaction: a randomized controlled trial study. Glob J Health Sci. 2014;6:219-226. positions. They should be encouraged to integrate the upright positions in their daily clinical practice. Further studies are needed to assess parturient women satisfaction toward the different birth positions, and evaluate the effect of upright position during the second stage of labor on the birth outcomes.

\section{Ethical approval}

This study was approved by the ethics committee of nursing college, Damanhour University (IRB approval number: 01-11-02-2018 EC). The researchers were committed to ethical rules at all study stages.

\section{Conflicts of interest}

There is no conflicts of interest.
9. Lawrence A, Lewis L, Hofmeyr GJ, Styles C. Maternal positions and mobility during first stage labour. Cochrane Database Syst Rev. 2013;10:1-10.

10. National Institute for Health and Care Excellence (UK). Intrapartum care for healthy women and babies. London; 2014. https://www.nice.org. uk/guidance/cg190/chapter/1-recommendations. Accessed October 14, 2019.

11. World Health Organization (WHO) Recommendation on adoption of mobility and upright position during labour in women at low risk (May 2014). The WHO Reproductive Health Library; Geneva: World Health Organization; 2014.

12. Gizzo S, GangiS, Noventa M, Veronaica, V, ZambonA, Nardelli G. Women's choice of positions during labor: return to the past or a modern way to give birth? A cohort study in Italy. Bio Med Res Int. 2014;Article ID 638093:1-7.

13. Bick D, Briley A, Brocklehurst $P$, et al. A multicentre, randomised controlled trial of position during the late stages of labour in nulliparous women with an epidural: clinical effectiveness and an economic evaluation. Health Technol Assess. 2017;21:1-176.

14. WHO. Partograph in management of labor. Lancet. 1994;343:1399-1404.

15. Apgar, V. A proposal for a new method of evaluation of the newborn infant. Curr Res Anesth Analg. 1953;32:260-267.

16. Mc Caffery M, Pasero C. Numeric Pain Rating Scale. Pain: Clinical Manual. 2nd ed. St. Louis, MO: Mosby; 1999:67. 
17. Iravani M, Janghorbani M, Zarean E, Bahrami M. An overview of systematic reviews of normal labor and delivery management. Iran J Nurs Midwifery Res. 2015;20:293-303.

18. Emam, AM, Al-Zahrani, AE. Upright versus recumbent position during first stage of labor among primipara women on labor outcomes. J Nurs Educ Pract. 2018;8:113-124.

19. Mathew A, Nayak S, Vandana K. A comparative study on effect of ambulation and birthing ball on maternal and newborn outcome among primigravida mothers in selected hospitals in Mangalore. NUJHS. 2012;2:2-5.

20. Mirzakhani K, Hejazinia Z, Golmakani N, Sardar MA, Shakeri MT. Effect of performing birth ball exercises during pregnancy on mode of delivery in primiparous women. J Midwifery Reprod Health. 2015;3:269-275.

21. Barbieri M, Henrique AJ, Chors FM, Maia NL, Gabrielloni MC. Warm shower aspersion, perineal exercises with Swiss ball and pain in labor. Acta Paul Enferm. 2013;26:478-484.

22. Hassan NZ. Effect of pelvic rocking exercise using sitting position on birth ball during the first stage of labor on its progress. IOSR J Nurs Health Sci. 2016;5:19-27.

23. Manonmani S. Effectiveness of Ambulation Versus Semi Fowler's Position on Labor Outcome among Parturient Mothers at Selected Hospital, Chennai.
Tamilnadu Dr. M.G.R Medical University: Chennai; 2016.

24. Miquelutti MA, Cecatti JG, Makuch MY. Upright position during the first stage of labor: a randomized controlled trial. Acta Obstet Gynecol Scand. 2007;86:553-558.

25. Rana KA, Chopra S. Effect of upright positions on the duration of first stage of labor among nulliparous mothers. Nurs Midwifery Res J. 2013;9:10-20.

26. Ben Regaya L, Fatnassi R, Khlifi A, et al. Role of deambulation during labour: a prospective randomized study. J Gynecol Obstet Biol Reprod (Paris). 2010;39:656-662.

27. Gau M, Chang C, Tian S, Lin K. Effects of birth ball exercise on pain and self-efficacy during childbirth. A randomized controlled trial in Taiwan. Midwifery. 2011;27:293-300.

28. Taavoni S, Abdolahian S, Haghani H, Neysani L. Effect of birth ball usage on pain in the active phase of labor: a randomized controlled trial. J Midwifery Women's Health. 2011;56:137-140.

29. Deliktaş A, Kabukçuoğlu K. The effect on childbirth types of upright positions during the first stage of labour: a meta-analysis study. Clin Exp Health Sci. 2018:8:128-137.

30. Roberts CL, Algert CS, Olive E. Impact of first-stage ambulation on mode of delivery among women with epidural analgesia. Aust N Z J Obstet Gynaecol. 2004;44:489-494. 